\title{
Special issue: Fractional signals and systems
}

\author{
Clara M. Ionescu • Jocelyn Sabatier • \\ J. A. Tenreiro Machado
}

Received: 15 March 2012 / Revised: 25 March 2012 / Accepted: 2 April 2012 / Published online: 4 May 2012

(C) Springer-Verlag London Limited 2012

Fractional order differentiation is a generalisation of classical integer differentiation to real or complex orders. From a mathematical point of view, several interpretations of fractional differentiation were proposed: all these interpretations demonstrate that fractional order differentiation cannot simply be connected to the local behaviour of the derivative function. This lack of simple interpretation is in fact due to the definition of the fractional order operator. This is a non-local operator based on an integral with a singular kernel. The same conclusion can be made for the fractional integrator operator, fractional differentiation operator definition being based on the fractional integrator operator definition. This situation explains why several definitions still coexist. Since the first recorded reference work in 1695 up to the present day, many articles have been published on this subject, but much progress still to be done particularly on the relationship of these different definitions with the physical reality of a system (through taking into account the initial conditions for instance).

A fractional order system is a system described by an integro-differential equation involving fractional order

\section{M. Ionescu ( $\square)$}

Department of Electrical Energy, Systems and Automation, Ghent University, Technologiepark 913, 9052 Gent-Zwijnaarde, Belgium e-mail: ClaraMihaela.Ionescu@UGent.be

\section{J. Sabatier}

IMS/LAPS: Automatique, Productique, Signal et Image, Université Bordeaux1-ENSEIRB-UMR 5218 CNRS, Bat A4-351, Cours de la Libération, 33405 Talence Cedex, France e-mail: jocelyn.sabatier@laps.ims-bordeaux.fr

\section{J. A. T. Machado}

Department of Electrical Engineering, Institute of Engineering of Polytechnic of Porto, Rua Dr. Antonio Bernardino de Almeida, 431, 4200-072 Porto, Portugal

e-mail: jtm@isep.ipp.pt derivatives of its input(s) and/or output(s). From a physical point of view, linear fractional derivatives and integrals order systems are neither conventional linear systems nor conventional distributed parameter systems. They are in fact halfway between these two classes of systems and are particularly suited for diffusion phenomena modelling. They also have been a modelling tool well suited to a wide class of phenomena with non-standard dynamic behaviour, and the applications of fractional order systems are now well accepted in the numerous fields.

Among all these applications field, Signal and Image Processing is certainly one field that has seen the least development. One aim of this special issue is thus to present some recent developments in the field, to show to the community the potential of fractional differentiation and fractional differentiation. As shown in this special issue, fractional differentiation can be applied to extend Fourier transform, or to improve signal and image filtering methods. Fraction filters implementation is thus an area also tackled in this special issue. The first applications of fractional differentiation in Signal and Image Processing have been completed since a considerable time. Hence, software tools are now available for researchers who wish to start in the field, and some of them are presented in this special issue. The research developments in the areas of fractional differentiation are based on well-defined mathematical concepts and some others still to be developed. Bearing these ideas in mind, recent mathematical developments are thus presented here. For the sake of openness, the modelling and control application fields are also addressed.

This special issue is only a sample of the work carried out throughout the world, but we hope it is representative of the envisaged themes and represents a useful source of information to begin with fractional differentiation and its applications. 
Fifteen papers have been selected out of a total of thirtyfour submissions. According to our previous comments and for presentation clarity, these papers have been gathered into three groups:

- Mathematical developments of fractional differentiation

- Signal processing and fractional filters implementation

- Fractional Modelling and Control applications
To conclude, we would like to thank the editorial board for allowing us to prepare this special issue and all the colleagues who have replied to our call for papers. 